\title{
Cortisol in Saliva and Plasma of Cattle After ACTH Administration and Milking
}

\author{
J. A. Negrão, ${ }^{1}$ M. A. Porcionato, ${ }^{2}$ A. M. de Passillé, ${ }^{3}$ and J. Rushen ${ }^{3}$ \\ ${ }^{1}$ Basic Science Department, USP/FZEA, University of São Paulo, 13630-970, \\ Pirassununga/SP, Brazil \\ ${ }^{2}$ UNESP, University of São Paulo State, 14884-900, Jaboticabal/SP, Brazil \\ ${ }^{3}$ Dairy and Swine Research and Development Centre, Agriculture and Agri-Food Canada, \\ Lennoxville, Quebec, J1M 1 Z3 Canada
}

\begin{abstract}
Interest in the measurement of salivary cortisol has increased recently because saliva can be easily collected before and after an imposed stress. This study evaluated the relationship between plasma and salivary concentrations of cortisol following ACTH administration in calves (experiment 1) and machine milking of adult cows (experiment 2). A catheter was inserted into the jugular vein of all animals $72 \mathrm{~h}$ before the beginning of experiments. Blood and saliva samples were collected before and after ACTH administration (0.6 IU/kg BW) in calves or before and after machine milking of cows. Using a cotton swab, each saliva sample was taken immediately following the blood sample. In general, cortisol profiles were similar in plasma and saliva and correlated in both experiments; however, plasma concentrations were significantly higher than salivary concentrations. In addition, the differences between cortisol concentrations measured in saliva and plasma within each experiment varied substantially between animals and samples. Furthermore, in experiment 2, nearly $10 \%$ of salivary samples were below limits of detection. The sharp peaks in cortisol after ACTH administration in both the plasma and saliva were reflected adrenal stimulation. In addition, increases in cortisol in response to milking in both the plasma and saliva suggest that salivary sampling is a reliable option when studying cortisol responses to normal physiological events.
\end{abstract}

(Key words: ACTH administration, cortisol, plasma, saliva)

\section{INTRODUCTION}

An increase in hypothalamic-pituitary-adrenocortical activity indicates a physiological response to differ-

Received July 10, 2003.

Accepted December 1, 2003.

Corresponding author: J. A. Negrão; e-mail: jnegrao@usp.br. ent stressors, and measurement of plasma corticosteroids is frequently used to study stress response (Sapolsky et al., 2000; Pacak and Palkovits, 2001). However, blood sampling itself is disturbing and the use of previous catheterization to minimize this disturbance is not always possible (Fell and Shutt, 1986; Cook et al., 1996). Consequently, there is interest in measuring corticosteroid concentrations in other body fluids, which would result in minimal stress (Möstl and Palme, 2002).

Although urine and fecal samples can be easily collected without submitting animals to stress (Palme et al., 1996), the volume/unit weight varies between animals and species (Palme et al., 1996), and time periods between application of the stressor and collection of the urine or feces cannot be controlled. Corticosteroids are typically metabolized soon after release. Palme et al. (1996) reported that cortisol is not (or only in trace amounts) present in fecal samples. As a result, corticosteroid metabolites must be measured in urine and feces.

In contrast, corticosteroids enter saliva by passive diffusion; thus, concentrations are unaffected by salivary flow rate (Riad-Fahmy et al., 1982). In addition, saliva samples can be easily taken at fixed time intervals before and after an imposed stress. For these reasons, interest in the measurement of salivary concentrations has increased during the past few years. Corticosteroid concentrations can be assayed in saliva, and measures of salivary cortisol concentrations have been used to assess stress reactions in cattle (Fell and Shutt, 1986; Cooper et al., 1989). Several authors have demonstrated that corticosteroid concentrations in saliva are directly related to those in plasma in humans, dogs, pigs, and domestic ruminants (Riad-Fahmy et al., 1982; Fell et al., 1985; Parrott et al., 1989; Vincent and Michell 1992; Cook et al., 1996; Cook, 2002). Others, however, have observed limitations in the extent to which salivary and plasma concentrations are related (Blackshaw and Blackshaw, 1989). Furthermore, little information is available on the relationship between concentrations of corticosteroids in saliva and plasma of cattle, especially in response to stressors at different ages. 
The objective of the present study was to examine the relationship between changes in cortisol concentrations in plasma and saliva of cattle following activation of the hypothalamic-pituitary-adrenocortical axis. In one experiment, we used ACTH injection to model severe stress. In a second experiment, we used machine milking as an example of a normal, physiological stimulus.

\section{MATERIALS AND METHODS}

The experiments were carried out at the Dairy and Swine Research and Development Centre in Lennoxville, Quebec, Canada. Experimental procedures were approved by the Lennoxville Institutional Animal Care Committee and complied with the requirements of the Canadian Council for Animal Care. Animals were housed and cared for according to accepted practices (Agriculture Canada, 1990).

\section{Experiment 1}

Thirteen 3-mo-old Holstein calves (BW $=125 \pm 20$ $\mathrm{kg})$ were housed in individual pens $(1.50 \times 2.10 \mathrm{~m})$ that permitted visual and vocal contact and limited tactile contact between neighboring calves. Pens were cleaned and bedded with wood shavings daily. The barn was maintained at 12 to $16^{\circ} \mathrm{C}$ and 70 to $80 \%$ humidity, and was illuminated by artificial light from 0530 to $1930 \mathrm{~h}$. Each calf received $2 \mathrm{~kg}$ of a mixed grain (Goliath Ration, Coopérative Féderée du Québec) at 0900 and $1500 \mathrm{~h}$ daily, and had free access to hay and water (from a nose-press water bowl).

A polyethylene catheter was inserted nonsurgically into the jugular vein of each calf $72 \mathrm{~h}$ before the beginning of the experiment. Between 0800 and $0830 \mathrm{~h}, 0.6$ IU injections of ACTH (Porcine ACTH 1-24, Sigma, St. Louis, MO), per kilogram of BW, were administered via the catheter (Fulkerson and Jamieson, 1982; Alan et al., 1986).

Blood and saliva samples were collected at $-40,-20$, and 0 min before, and at 20,40,60, 120, 180, 240, 300, and 360 min after ACTH injection. Each saliva sample (up to $2 \mathrm{~mL}$ ) was taken immediately after each blood samples (approximately $10 \mathrm{~mL}$ ). Blood samples required $0.5 \mathrm{~min}$, and saliva samples required up to 3 min to collect. Saliva samples were collected on cotton swabs held manually in a pair of surgical clamps and placed manually into the animal's mouth.

\section{Experiment 2}

Six Holstein dairy cows, between 3 and 4 yr of age and at $145 \pm 15 \mathrm{~d}$ of lactation, were housed and milked (at 0600 and $1800 \mathrm{~h})$ in individual tie stalls $(1.30 \times 1.95$ $\mathrm{m})$. Milking routines were constant performed by the same person. The following machine parameters were constant throughout the experiment: vacuum $=38 \mathrm{kPa}$, pulsation ratio $=60: 40 \%$, and pulsation rate $=60 \mathrm{c} /$ min. The cows produced $27.0 \pm 2.3 \mathrm{~kg}$ of milk per day. They received a TMR (mixed grain, silage and hay) at 0900 and $1400 \mathrm{~h}$ and had free access to water (from a nose-press water bowl).

A polyethylene catheter was inserted into the jugular vein of each experimental animal $72 \mathrm{~h}$ before the beginning of the experiment. For 2 consecutive mornings, blood, and saliva samples were collected at $-40,-20$, and 0 min before and at 10,20,40,60,120, and 360 min after machine milking. Saliva samples were taken immediately after each blood sample as described for experiment 1.

\section{Cortisol Assay}

Blood was collected in heparinized tubes, placed on ice, and then centrifuged at $4^{\circ} \mathrm{C}$ and $3000 \times g$ for 15 min. Cotton swabs were inserted into a plastic syringe and the piston was depressed to collect $2 \mathrm{~mL}$ of saliva in normal test tubes, which were placed on ice immediately after sampling. One milliliter of saliva was lyophilized to concentrate the samples. The tubes containing plasma or lyophilized saliva were then sealed and stored at $-20^{\circ} \mathrm{C}$ until cortisol determination using an enzyme immunoassay kit (Neogen, Lexington, KY). Lyophilized saliva was first dissolved in $200 \mu \mathrm{L}$ of physiological solution, and then $100 \mu \mathrm{L}$ of plasma or saliva was added to $1 \mathrm{~mL}$ of ethyl ether and vortexed for 0.5 min to separate the organic phase, which was then pipetted into a tube and evaporated with $\mathrm{N}_{2}$. The residue was dissolved in extraction buffer $(200 \mu \mathrm{L})$. Standards, extracted samples ( $50 \mu \mathrm{L})$, enzyme solution and antiserum were added on the plates. After incubation, the plate was washed, substrate was added, and the plate was read at $650 \mathrm{~nm}$. All samples were tested in duplicate; samples were reassayed if duplicates differed by more than $10 \%$. Interassay CV were 8.11 and $9.15 \%$ for plasma and saliva, respectively, and intraassay CV were 9.26 and $9.54 \%$ for plasma and saliva, respectively. The kit was validated by demonstrating parallel curves between standard concentrations and serially diluted plasma and saliva samples (Figure 1).

\section{Statistical Analysis}

The SAS software package (SAS Inst., Inc., Cary, NC) was used for analysis of data by means of ANOVA, based on mixed models, Student's $t$-tests, and NewmanKeuls tests. Significance was set at $P<0.05$, and all 


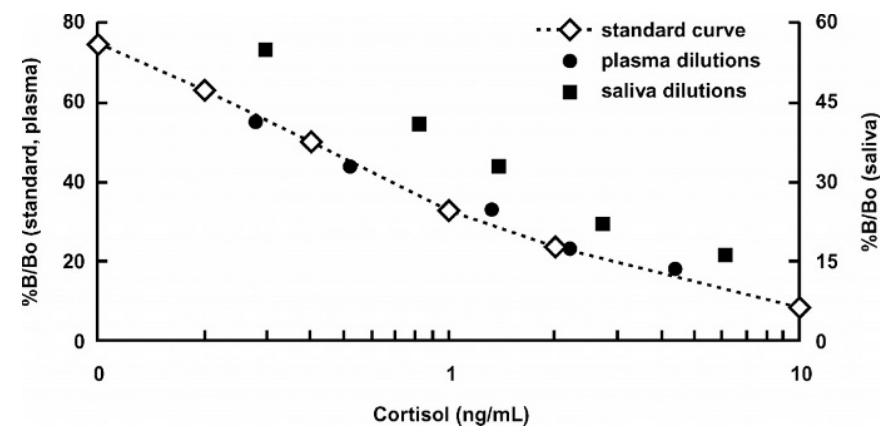

Figure 1. Parallelism curves between standard curve $(\diamond)$, plasma $(\bullet)$, and saliva (ם) serially diluted.

values are given as the mean \pm one standard error of the mean (SEM). The relationship between cortisol measured in plasma and saliva samples was evaluated by Pearson correlation coefficients. The statistical model used to evaluate the effect of treatment on cortisol levels had a stimulus (ACTH administration or machine milking) as the main factors, and day and time of sampling as repeated measures, and included all possible interactions between factors. The model performed for each experiment was as follows:

Experiment 1 (ACTH administration)

$$
\mathrm{Y}_{\mathrm{ij}}=\mu+\mathrm{Cal}_{\mathrm{i}}+\mathrm{T}_{\mathrm{j}}+\text { error }_{\mathrm{ij}}
$$

where $Y_{i j}=$ cortisol level (in plasma or saliva), $\mu=$ the overall mean, $\mathrm{Cal}_{\mathrm{i}}=$ effect of calf $\mathrm{i}(\mathrm{i}=1,2,3, \ldots, 13)$, $\mathrm{T}_{\mathrm{j}}=$ effect of sampling time $\mathrm{j}(\mathrm{j}=-40,-20,0,20 \ldots$, $360)$, and error $_{i j}=$ residual variation.

Experiment 2 (milking machine)

$$
\mathrm{Y}_{\mathrm{ijk}}=\mu+\mathrm{Cow}_{\mathrm{i}}+\mathrm{D}_{\mathrm{j}}+\mathrm{T}_{\mathrm{k}}+\mathrm{D}_{\mathrm{j}} \mathrm{T}_{\mathrm{k}}+\text { error }_{\mathrm{ijk}}
$$

where $\mathrm{Y}_{\mathrm{ijk}}=$ cortisol levels (in plasma or saliva), $\mu=$ the overall mean, $\mathrm{Cow}_{\mathrm{i}}=$ effect of cow $\mathrm{i}(\mathrm{i}=1,2,3, \ldots$ ., 6), $\mathrm{D}_{\mathrm{j}}=$ effect of day $\mathrm{j}(\mathrm{j}=1,2), \mathrm{T}_{\mathrm{k}}=$ effect of sampling time $\mathrm{k}(\mathrm{k}=-40,-20,0,10,20 \ldots, 360), \mathrm{D}_{\mathrm{j}} \mathrm{T}_{\mathrm{k}}=$ effect of sampling time $\mathrm{k}(\mathrm{k}=-40,-20,0,10,20 \ldots, 360)$ within day $\mathrm{j}(\mathrm{j}=1,2)$, and error $\mathrm{ij}_{\mathrm{ij}}=$ residual variation.

\section{RESULTS}

\section{Experiment 1}

Cortisol levels measured before and after ACTH injections in calves are shown in Figure 2A. Compared to baseline (samples $-40,-20$, and $0 \mathrm{~min}$ ), plasma and saliva cortisol increased significantly after ACTH ad- ministration to reach their maximal levels at $40 \mathrm{~min}$. Afterward, a gradual decrease in cortisol concentration was observed in both fluids, but concentrations were still significantly higher than basal levels at $120 \mathrm{~min}$. However, cortisol levels measured at $360 \mathrm{~min}$ not differ from basal levels. Although cortisol levels were substantially higher in plasma than in saliva, the two profiles were similar and there was a significant and positive correlation between salivary and plasma concentrations $(\mathrm{r}=0.71, \mathrm{n}=192, P<0.01)$.

\section{Experiment 2}

Cortisol concentration before and after machine milking are shown in Figure 2B. In both fluids, cortisol concentration increased rapidly and significantly at the beginning of milking machine and peaked at $20 \mathrm{~min}$. Cortisol concentrations decreased once milking was finished to near basal levels at $120 \mathrm{~min}$. In general, plasma levels were substantially higher than in saliva (Figure 2B). Again, similar profiles existed in the two fluids and there was a significant, positive correlation between salivary and plasmatic concentrations across samples $(\mathrm{r}=0.63, \mathrm{n}=108, P<0.01)$.

Within each experiment, the difference between cortisol measured in saliva and plasma varied substantially between individuals and between samples (Figure 3). However, it was possible to classify the response to ACTH administration (Figure 3A) or machine milking (Figure 3B) as high or low for certain animals based on the integrated surface under the cortisol curves corrected for basal levels. These extreme responses were consistent using salivary or plasma concentrations.

\section{DISCUSSION}

When compared to basal concentrations, cortisol concentrations increased significantly following ACTH administration and milking machine. In general, our results were similar to those observed by other authors in calves after ACTH administration (Veissier and Le Neidre, 1988; Veissier et al., 1999) and in cows during and after machine milking (Wagner and Oxenreider, 1972; Gorewit et al., 1992; Rushen et al., 2001). These responses were consistently found in both plasma and salivary concentrations of cortisol, although the response was substantially greater for plasma. A similar result has been previously noted in pigs (Parrott et al., 1989) and dogs (Vincent and Michell, 1992).

For both plasma and saliva, the cortisol response was significantly lower after milking than after ACTH administration. This confirms the concept that a maximal response can be obtained with the ACTH dose used (Bremel and Gangwer, 1978; Safwate et al., 1982; Lay 


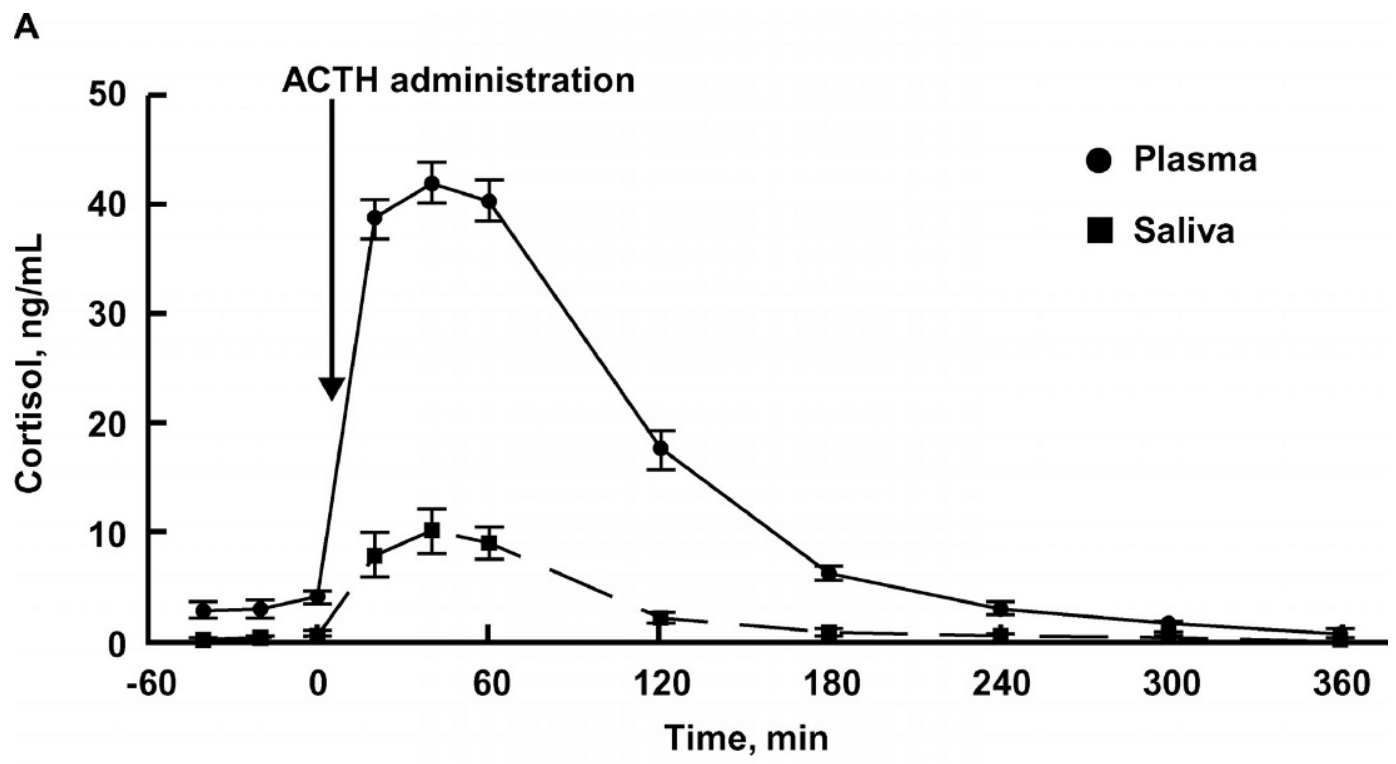

B

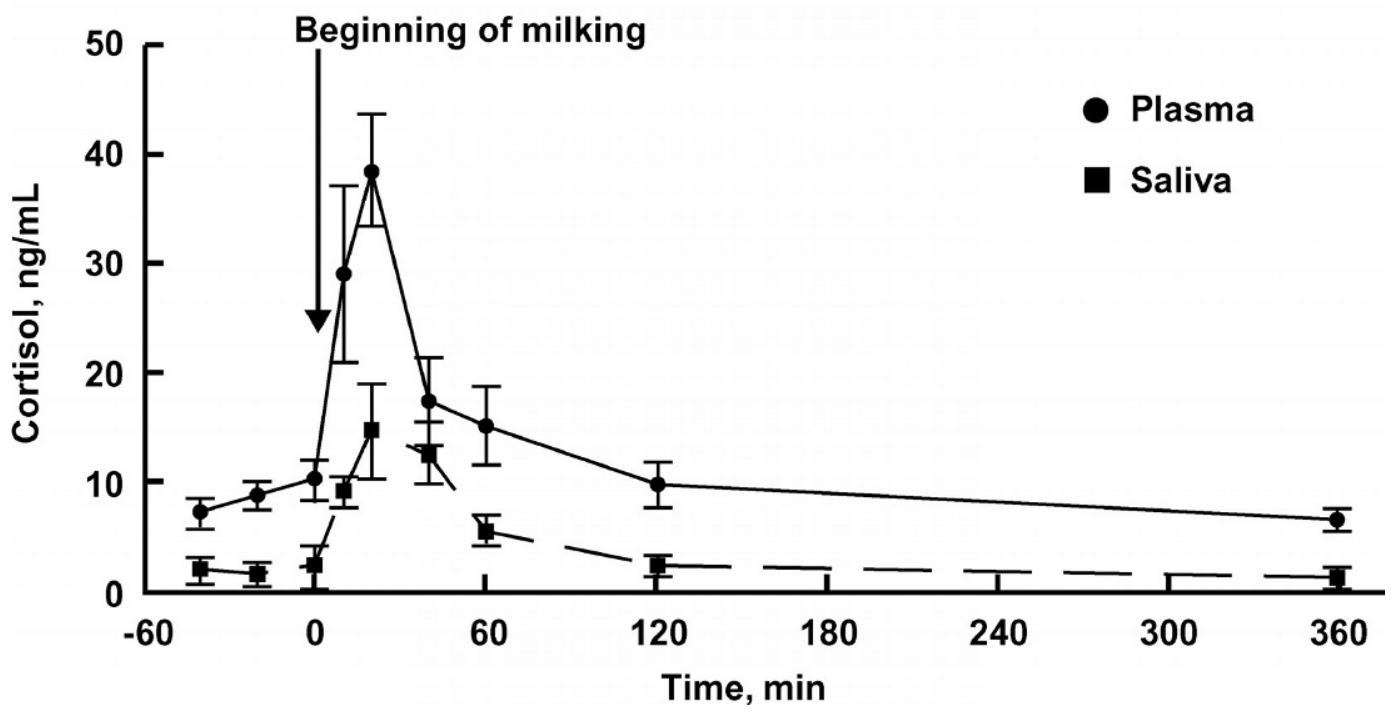

Figure 2. Cortisol levels $(\mathrm{ng} / \mathrm{mL})$ in plasma $(\bullet)$ and saliva ( $)$ before and after ACTH administration in calves (A) or machine milking in cows (B). Values are means and standard errors of the mean.

et al., 1996; Veissier et al., 1999), whereas the response caused by milking is small (Tancin et al., 1995; Rushen et al., 2001). As previously observed by other authors, usually only severe stressors are found to have effects on cortisol concentrations similar to those measured after ACTH administration (Fulkerson and Jamieson, 1982; Lay et al., 1996).

In blood, the assay measures total cortisol concentration (free fraction plus bond fraction), but this may not necessarily reflect the biological action of the hormone. In saliva, the cortisol concentration that is measured is a direct reflection of the free fraction in the blood (Riad-Fahmy et al., 1982). Furthermore, under stress conditions, the binding capacity of cortisol-binding protein becomes saturated, resulting in a disproportionate increase in free cortisol, the fraction that is biologically active (Cook et al., 1997). These facts partly explain the differences between concentrations of plasma and salivary cortisol.

Concentrations of cortisol in saliva were substantially lower than those found in plasma, and this sometimes produced sampling problems. Although we concentrated the saliva samples 10 -fold, some cortisol concentrations in the samples remained lower than the minimal concentrations measured by the kit that was used. Consequently, $9.25 \%$ of salivary samples in exper- 
A

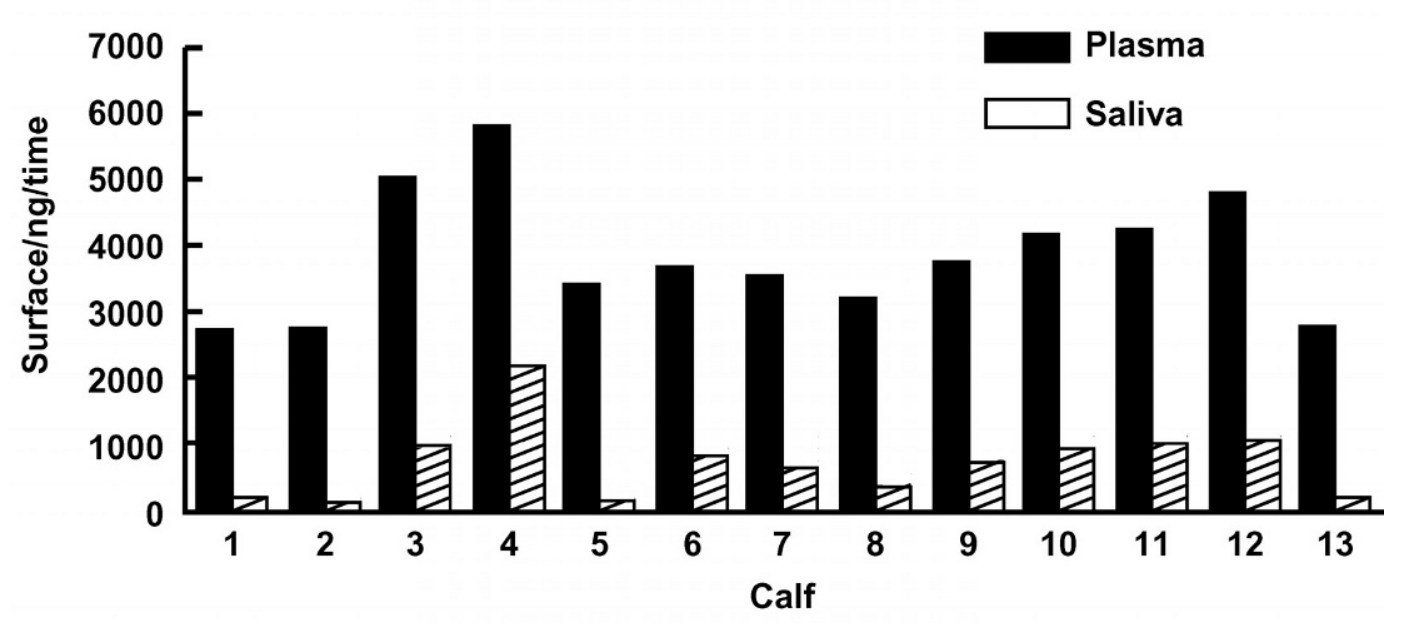

B

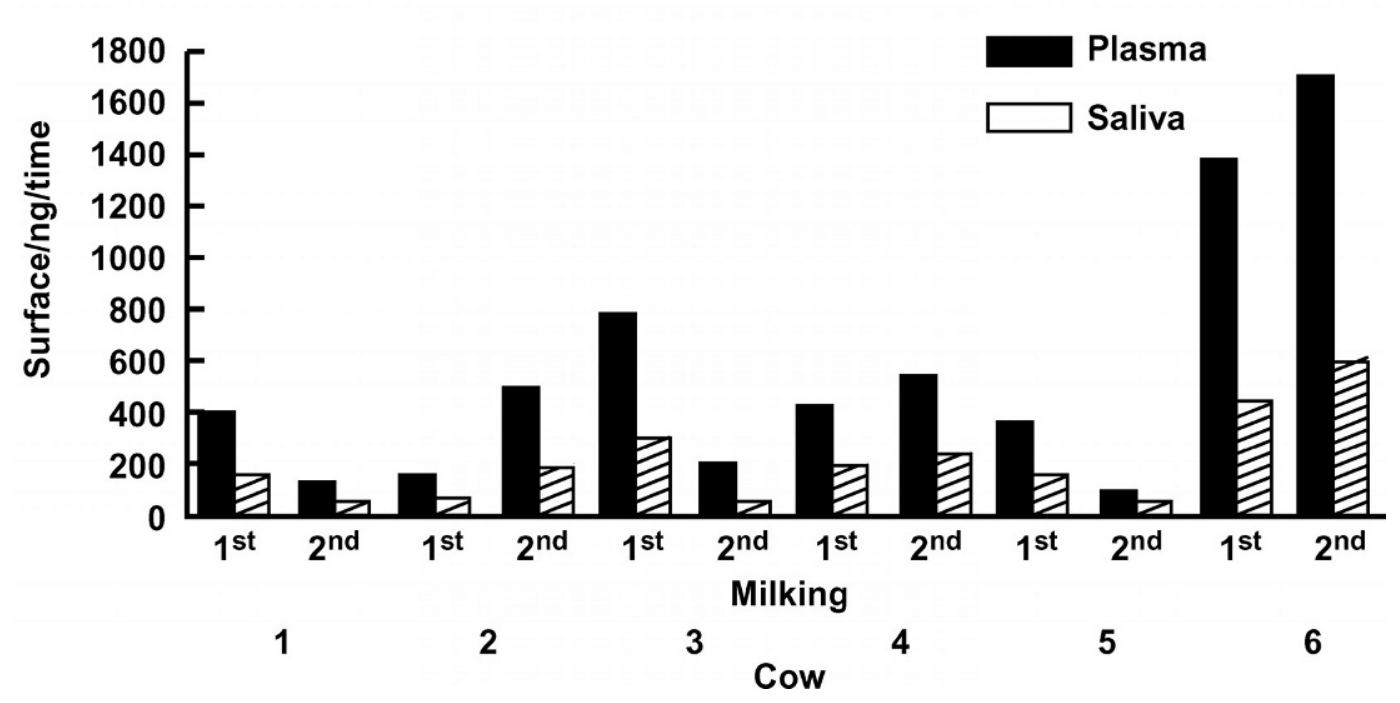

Figure 3. Total amount of cortisol released in individual animals after ACTH administration in calves (A) or machine milking in cows (B) for plasma (filled bars) and saliva (hatched bars). Total amount: based on all samples taken, the value of the integrated area under the curve corrected for the basal levels. Levels in cows were measured the first (1st) and second (2nd) morning of milking during 2 consecutive days.

iment 2 could not be measured. Unfortunately, the volume of saliva collected (approximately $2 \mathrm{~mL}$ ) was not sufficient to reassay the samples. This limitation could be reduced by greater concentration of samples but when frequent sampling is necessary, it is not possible to take a large volume of saliva in calves. Indeed, the calves did not produce saliva easily after 4 or 5 samples in this experiment. Other authors have noted similar concerns (Coste et al., 1994; Cook et al., 1996; 1997). However, our results suggest that measurement of cortisol in saliva may be reliably used to assess the re- sponse of cattle to both severe stress as well as physiological events, such as milking.

In general, it was easy to collect saliva from experimental calves because they readily sucked the cotton swab that was placed in their mouths. However, it was also necessary to manually restrain the calf's head. In contrast, it was substantially more difficult to take saliva samples from adult cows. Although the cows remained tied in tie stalls throughout the experiment, it was necessary to restrain the cows' heads with a halter and to hold their mouths open. Furthermore, it took 
substantially more time to collect saliva samples than blood samples ( 3 min vs. 30 s). For these reasons, we suggest that saliva sampling should not be considered a stress-free method of sampling, particularly in adult cows. However, the peaks in cortisol levels observed in the plasma and saliva of cows were not due to the sampling procedures because the cortisol levels measured in experiment 2 were very similar to those obtained during normal machine milking (Gorewit et al., 1992; Rushen et al., 2001), and were too low to be the result of severe stress. Consequently, blood sampling $72 \mathrm{~h}$ after catheterization and saliva sampling can be considered techniques that are not highly stressful.

\section{CONCLUSIONS}

The significant positive correlations between salivary and plasmatic concentrations and the parallel responses to ACTH and milking show that it is possible to estimate cortisol concentrations in the plasma from salivary concentrations in both calves and adult cattle. The increase in cortisol concentration in response to ACTH administration or machine milking are representative of adrenal activity or milking stimulus, respectively.

\section{ACKNOWLEDGMENTS}

Funding was received from the Dairy Cattle Genetics Research and Development Council of Canada. J. A. Negrão and M. A. Porcionato were supported by CAPES fellowships. We thank B. van Doormaal for his support, D. Glimm for discussion, I. Blanchet, M. St. Louis, and M.-J. Sirois for excellent technical help, and the staff of the Lennoxville Dairy Centre for their assistance.

\section{REFERENCES}

Agriculture Canada. 1990. Recommended Cod of Practice for the Care and Handling of Cattle. Agriculture Canada, Ottawa.

Alan, M. G. S., H. Dobson, and R. J. Fitzpatrick. 1986. Endocrine response to different doses of ACTH in cows. Br. Vet. J. 142:239-245.

Blackshaw, J. K., and A. W. Blackshaw. 1989. Limitations of salivary and blood cortisol determinations in pigs. Vet. Res. Commun. 13:265-271.

Bremel, R. D., and M. I. Gangwer. 1978. Effect of adrenocorticotropin injection and stress on milk cortisol content. J. Dairy Sci. 61:1103-1108.

Cook, C. J. 2002. Rapid noninvasive measurement of hormones in transdermal exudate and saliva. Physiol. Behav. 75:169-181.

Cook, N. J., A. L. Schaefer, P. Lepage, and S. Morgan Jones. 1996. Salivary vs. serum cortisol for the assessment of adrenal activity in swine. Can. J. Anim. Sci. 76:329-335.
Cook, N. J., A. L. Schaefer, P. Lepage, and S. Morgan Jones. 1997. Radioimmunoassay for cortisol in pig saliva and serum. J. Agric. Food Chem. 45:395-399.

Cooper, T. R., H. R. Trunkfield, A. J. Zanella, and W. D. Booth. 1989. An enzyme-linked immunosorbent assay for cortisol in the saliva of man and domestic farm animals. J. Endocrinol. 123:R13-R16.

Coste, J., G. Strauch, M. Letrait, and X. Bertagna. 1994. Reliability of hormone levels for assessing the hypothalamic-pituitary-adrenal system in clinical pharmacology. Br. J. Clin. Pharmacol. 38:474-479.

Fell, L. R., and D. A. Shutt. 1986. Adrenocortical response of calves to transport stress as measured by salivary cortisol. Can. J. Anim. Sci. 66:637-641.

Fell, L. R., D. A. Shutt, and C. J. Bentley. 1985. Development of a salivary cortisol method for detecting changes in plasma "free" cortisol arising from acute stress in sheep. Aust. Vet. J. 12:403-406.

Fulkerson, W. J., and P. A. Jamieson. 1982. Pattern of cortisol release in sheep following administration of synthetic ACTH or imposition of various stressor agents. Aust. J. Biol. Sci. 35:215-222.

Gorewit, R. C., K. Svennersten, W. R. Butler, and K. Uvnas-Moberg. 1992. Endocrine responses in cows milked by hand and machine. J. Dairy Sci. 75:443-448.

Lay, Jr., D. C., T. H. Friend, R. D. Randel, O. C. Jenkins, D. A. Neuendorff, G. M. Kapp, and D. M. Bushong. 1996. Adrenocorticotropic hormone dose response and some physiological effects of transporation on pregnant Brahman cattle. J. Anim. Sc. 74:1806-1811.

Möstl, E., and R. Palme. 2002. Hormones as indicators of stress. Domest. Anim. Endocrinol. 23:67-74.

Pacak, K., and M. Palkovits. 2001. Stressor specificity of central neuroendocrine responses: implications for stress-related disorders. Endocrinol. Rev. 22:502-548.

Palme, R., P. Fischer, H. Schildorfer, and M. N. Ismail. 1996. Excretion of infused C-steroid hormones via faeces and urine in domestic livestock. Anim. Reprod. Sci. 43:43-63.

Parrott, R. F., B. H. Misson, and B. A. Baldwin. 1989. Salivary cortisol in pigs following adrenocotrophic hormone stimulation: comparison with plasma levels. Br. Vet. J. 145:362-366.

Riad-Fahmy, D., G. F. Read, R. F. Walker, and K. Griffiths. 1982. Steroids in saliva for assessing endocrine function. Endocrinol. Rev. 4:367-395.

Rushen, J., L. Munksgaard, P. G. Marnet, and A. M. De Passillé. 2001. Human contact and the effects of acute stress on cows at milking. Appl. Anim. Behav. Sci. 73:1-14.

Safwate, A., E. Richet, J. P. Barlet, and P. Delost. 1982. ACTH and plasma corticosteroid levels in young calves. J. Physiol. 78:195-197.

Sapolsky, R. M., M. L. Romero, and A. U. Munck. 2000. How do glucocorticoids influence stress responses? Integrating permissive, suppressive, stimulatory, and preparative actions. Endocrinol. Rev. 21:55-89.

Tancin, V., L. Harcek, J. Broucek, M. Uhrincat, and S. Mihina. 1995. Effect of suckling during early lactation and changeover to machine milking on plasma oxytocin and cortisol levels and milking characteristics in Holstein cows. J. Dairy Res. 62:249-256.

Veissier, I., and P. Le Neindre. 1988. Cortisol responses to physical and pharmacological stimuli in heifers. Reprod. Nutr. Dev. 28:553-562.

Veissier, I., C. G. van Reenen, S. Andanson, and I. E. Leushuis. 1999. Adrenocorticotropic hormone and cortisol in calves after corticotropin-releasing hormone. J. Anim. Sci. 77:2047-2053.

Vincent, I. C., and A. R. Michell. 1992. Comparison of cortisol concentrations in saliva and plasma of dogs. Res. Vet. Sci. 53:342-345.

Wagner, W. C., and S. L. Oxenreider. 1972. Adrenal function in the cow: Diurnal changes and the effects of lactation and neurohypophyseal hormones. J. Anim. Sci. 34:630-635. 